\title{
Distribution and chemical coding pattern of somatostatin immunoreactivity in the dorsal striatum of the guinea pig
}

\author{
Barbara Wasilewska ${ }^{1}$, Anna Robak ${ }^{1}$, Maciej Równiak ${ }^{1}$, Krystyna Bogus-Nowakowska1, \\ Janusz Najdzion', Witold Żakowski', Mariusz Majewski² \\ ${ }^{1}$ Department of Comparative Anatomy, Faculty of Biology, University of Warmia and Mazury \\ in Olsztyn, Poland \\ ${ }^{2}$ Department of Human Physiology, Faculty of Medicine, University of Warmia and Mazury \\ in Olsztyn, Poland
}

\begin{abstract}
The present study provides a detailed description of somatostatin (SOM) distribution and the colocalization pattern of SOM, neuropeptide Y (NPY) and nitric oxide synthase (NOS) in the dorsal striatum (caudate-putamen complex) of the guinea pig. Within the dorsal striatum, SOM is found in a population of medium-sized aspiny interneurons. We found that $97 \%$ of all SOM-IR neurons expressed NPY simultaneously, while $98 \%$ of all NPY-ergic perikarya was simultaneously SOM-IR. On the other hand, while $98 \%$ of all SOM-IR cells were simultaneously NOS-IR, only $91 \%$ of all NOS-containing neurons exhibited SOM-immunoreactivity. Irrespective of their chemical coding, both types of SOM-IR neurons were scattered throughout the dorsal striatum, sometimes in the form of small, loosely arranged clusters of 2-4 cells. While SOM-IR and NPY-IR nerve fibers were present in all of the studied regions, they were more numerous in the ventro-medial part of the studied structure, with the exception of its caudal portion, where SOM-IR and NPY-IR fibers additionally formed a dense network in the part corresponding to the caudate nucleus. A low expression of staining for NOS-IR fibers was seen throughout the entire dorsal striatum. In some fibers, SOM and NPY were co-expressed. Fibers expressing both SOM and NOS were not found. (Folia Histochemica et Cytobiologica 2011; Vol. 49, No. 4, pp. 690-699)
\end{abstract}

Key words: somatostatin, nitric oxide synthase, neuropeptide Y, dorsal striatum, guinea pig

\section{Introduction}

Somatostatin (SOM) is a cyclic tetradecapeptide that is isolated from the ovine hypothalamus [1]. SOM-positive nerve cell bodies and fibers are distributed widely throughout the central nervous system $[2,3]$. Within the dorsal striatum (the caudate-putamen complex), SOM is found in a population of medium-

\footnotetext{
Correspondence address: B. Wasilewska, Department of Comparative Anatomy, Faculty of Biology,

University of Warmia and Mazury in Olsztyn,

Plac Lodzki 3, 10-767 Olsztyn, Poland;

tel.: (+ 48 89) 5233602 ;

e-mail: bachaw@uwm.edu.pl
}

-sized aspiny interneurons [3]. SOM-immunoreactive neurons are involved in the feedforward inhibition of the spiny projection neurons, the modulation of other interneurons and the regulation of regional blood flow [4]. This population of neurons plays an important role in the processing of information in the dorsal striatum [4].

The dorsal striatum contains at least four major types of interneurons [5]: those co-containing GABA (gamma aminobutyric acid) and calretinin; those co-containing GABA and parvalbumin; those co-containing GABA and SOM, neuropeptide Y (NPY), and neuronal nitric oxide synthase (nNOS); and those containing acetylcholine (ACh) [6-10]. The type co-containing SOM, NPY and nNOS/NADPH-d 
(enzyme nicotinamide adenine dinucleotide phosphate diaphorase) makes up about $1 \%$ of all striatal neurons [11].

The morphology and chemical coding of SOM-IR neurons in the dorsal striatum has mostly been studied in rats [2, 3, 12-15]. A smaller amount of data has come from cats [16], hedgehogs and sheep [17], monkeys [18, 19] and humans [20, 21]. There has been only one short report concerning the morphology and chemical coding of SOM-IR neurons in the dorsal striatum of guinea pigs [22]. Vincent et al. [22] did not carry out any quantitative studies of the dorsal striatum in this animal.

The aim of our study was to describe the distribution and co-localization pattern of SOM and NPY or NOS in the dorsal striatum of this species.

SOM is a multifunctional peptide involved in several neurodegenerative diseases [23]. We have chosen only this pattern of co-localization because the neurons that contain SOM, NPY and NOS are relatively resistant to destruction in Huntington's disease (HD) and to destruction by quinolinic acid, at least under some conditions [24-27].

We hope that the results of this work may be useful in interpreting studies on the effects of neurodegenerative diseases, excitotoxins and other experimental manipulations on the survival of striatal neurons containing SS, NPY and/or NOS. These studies are particularly valuable because the guinea pig is more and more frequently used as a model in neuroscience and medical research $[28,29]$.

\section{Material and methods}

Animals and tissue specimen collection. Five sexually immature female guinea pigs (strain: Dunkin-Hartley, approximately 12 weeks old, obtained from the Research Institute of the Polish Mothers' Health Center in Lodz, Poland) were used in the present study. All experiments were carried out in accordance with the Local Ethical Committee rules. All efforts were made to minimize animal suffering and to use the minimum number of animals necessary to produce reliable statistical data. All animals were anesthetized with a lethal dose of sodium pentobarbital (Morbital, Biowet, Poland; $2 \mathrm{ml} / \mathrm{kg} \mathrm{b.w)} \mathrm{and} \mathrm{perfused} \mathrm{intracardially} \mathrm{with} 4 \%$ paraformaldehyde. Next, the brains were removed from the skulls and postfixed for 30 minutes in the same fixative, washed twice in $0.1 \mathrm{M}$ phosphate buffer and then cryoprotected in sucrose until they sank to the bottom of their container. Frozen brains were cut into $10 \mu \mathrm{m}$ coronal plane sections on a cryostat.

Immunohistochemistry. The sections were processed for routine single- and double-labeling immunofluorescence studies using a rat polyclonal antibody against SOM (1:600; 8330-0009, Biogenesis, UK) that was combined with one of the following rabbit polyclonal antisera against: NPY (1:2,000; NA1233, Affinity, UK) and NOS (1:2,000, B220-1, Euro-Diagnostica, Sweden). To visualize the antigen-antisera binding sites, the sections were incubated $(1 \mathrm{~h}$, at room temperature) with a mixture of Cy3-conjugated donkey anti-rat (1:6,000; 712-165-153, Jackson ImmunoLabs, USA) and FITC-conjugated donkey anti-rabbit (1:600; 711-095-152, Jackson ImmunoLabs, USA). To prove the specificity of immunoreaction, the control sections underwent replacement, omission, and cross-reaction. All control immunostainings showed no immunoreactivity. The sections were coverslipped in buffered carboxyglycerol.

Data analysis. The sections were viewed under an Olympus BX51 microscope. The images were captured with Cell B software (Soft Images Systems GmbH, Germany). The proportions of SOM-IR neurons co-expressing (or not) NPY or NOS were presented as a percentage relative to the total number of neurons analyzed.

Cell counts of single-labeled and double-labeled neurons were made in the whole dorsal striatum and were pooled from five animals. At $200 \times$ magnification, cell counts were made from the image of a $400 \times 400 \mu \mathrm{m}$ field displayed for merged (red/green) channels on the computer screen (double-labeled cells appear yellow). Images of the non-merged red and green channel were also displayed.

\section{Results}

\section{Structure of the guinea pig dorsal striatum}

In the guinea pig, the dorsal striatum takes the form of a caudate nucleus-putamen complex $(\mathrm{CPu})$ (Figures 1A, D, G). As in other mammalian species, the appearance of this complex depends on the general location of the fibers of the internal capsule (I). These fibers are found throughout the whole structure in the form of dispersed, oval structures (Figure 1D). At the level where the anterior commissure crosses the midline, the fibers are distinctly elongated in the dorso-ventral direction and the caudate nucleus slowly starts to separate from the complex. The two parts of the dorsal striatum separate fully where the ventral hippocampal commissure appears (Figure 1G). The caudate nucleus disappears earlier than the putamen.

The general cellular structure of the dorsal striatum in the guinea pig closely resembles its structure in the common shrew and water shrew [30]. In the present study, the dorsal striatum in the guinea pig was examined to determine the distribution and morphology of SOM-IR perikarya and fibers, and the colocalization of SOM with NPY and with NOS. 

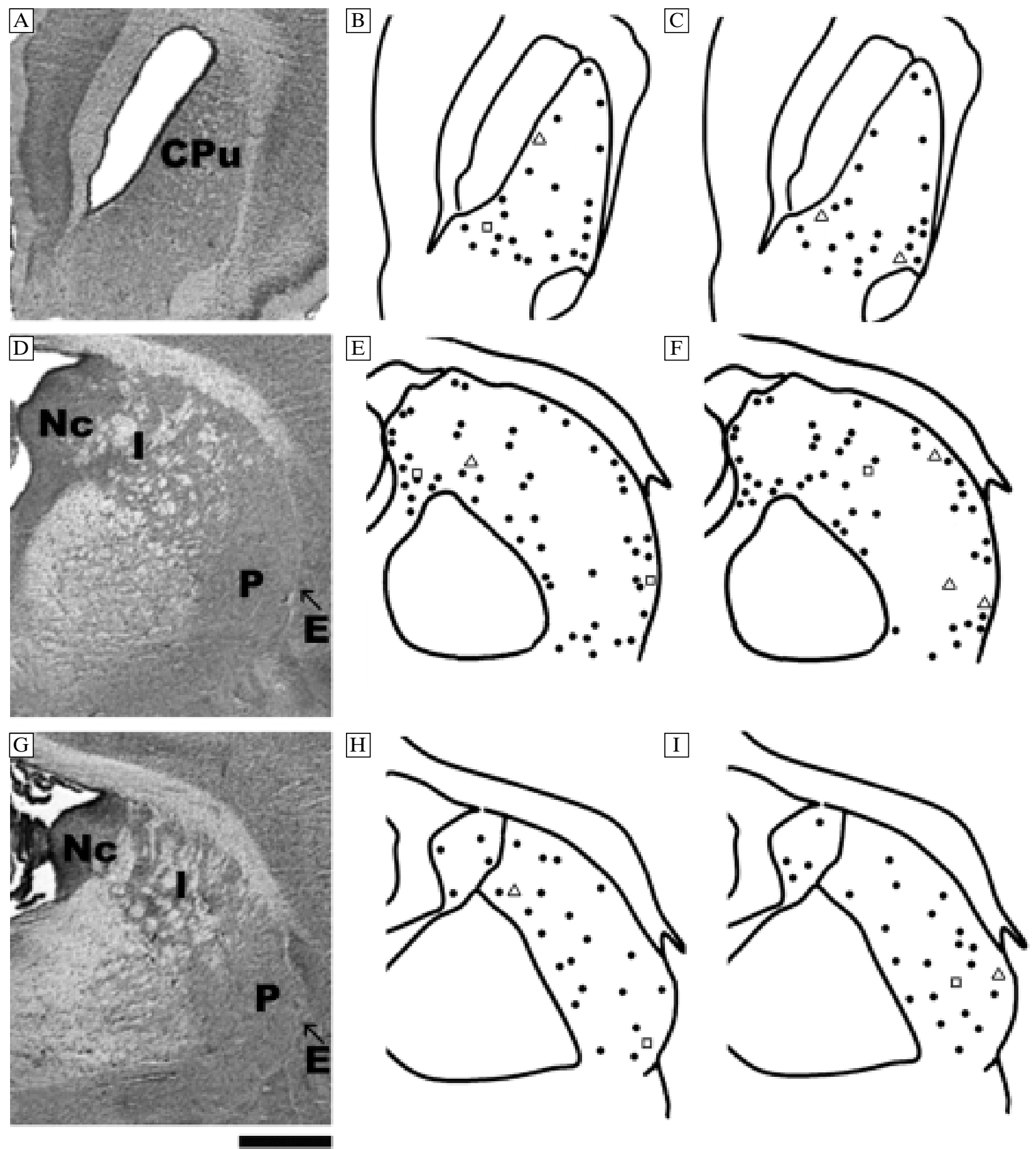

Figure 1. Microphotographs of Nissl-stained sections (A, D, G) and schematic drawings showing the co-localization pattern of SOM and NPY (B, E, H) as well as SOM and NOS (C, F, I) through the anterior (A-C), middle (D-F) and caudal (G-I) portions of the guinea pig dorsal striatum. $\mathrm{CPu}$ - dorsal striatum; E - external capsule; I - internal capsule; Nc - caudate nucleus; P — putamen; Dots - SOM/NPY (B, E, H) or SOM/NOS (C, F, I); squares - SOM (B, E, F, H, I); triangles — NPY (B, E, H) or NOS (C, F, I). Scale bar: $1 \mathrm{~mm}$

\section{Distribution pattern and morphology of SOM-, NPY-, and NOS-structures in the dorsal striatum}

The distribution pattern of the SOM-, NPY- and NOS-immunoreactive structures within the guinea pig dorsal striatum is shown in Figures 1B, C, E, F, H, and I and Tables 1-2. The morphology and characteristic features of the whole structure are shown in Figures 2-3.

All the antibodies that were used produced intensive immunohistochemical staining in the dorsal striatum and several other forebrain areas. The patterns of immunohistochemical staining and the frequency 
Table 1. Numbers and relative frequencies of particular subclasses of dorsal striatal neurons stained simultaneously for SOM and NPY (a) as well as SOM and NOS (b)

\begin{tabular}{|c|c|c|c|c|c|c|}
\hline & $\begin{array}{c}\text { Total } \\
\text { neurons } \\
\text { counted }\end{array}$ & $\mathbf{S O M}^{+} / \mathbf{N P Y}^{+}$ & $\mathbf{S O M}^{+} / \mathbf{N P Y}^{-}$ & $\mathbf{S O M}^{-} / \mathbf{N P Y}^{+}$ & $\begin{array}{c}\text { Percentage } \\
\text { of SOM-IR neurons } \\
\text { containing NPY }\end{array}$ & $\begin{array}{c}\text { Percentage } \\
\text { of NPY-IR neurons } \\
\text { containing SOM }\end{array}$ \\
\hline a) SOM + NPY & 4,300 & 4,104 & 128 & 68 & 97.0 & 98.4 \\
\hline & $\begin{array}{c}\text { Total } \\
\text { neurons } \\
\text { counted }\end{array}$ & $\mathbf{S O M}^{+} / \mathbf{N O S}^{+}$ & $\mathbf{S O M}^{+} / \mathbf{N O S}^{-}$ & $\mathbf{S O M}^{-} / \mathbf{N O S}^{+}$ & $\begin{array}{c}\text { Percentage } \\
\text { of SOM-IR neurons } \\
\text { containing NOS }\end{array}$ & $\begin{array}{c}\text { Percentage } \\
\text { of NOS-IR neurons } \\
\text { containing SOM }\end{array}$ \\
\hline b) SOM + NOS & 4,851 & 4,353 & 75 & 423 & 98.3 & 91.1 \\
\hline
\end{tabular}

Table 2. Distribution and density patterns of SOM, NPY and NOS fibers in the anterior, middle and caudal portions of the guinea pig dorsal striatum

\begin{tabular}{|l|c|c|c|c|c|c|}
\hline \multirow{2}{*}{} & \multicolumn{2}{|c|}{ Anterior portion } & \multicolumn{2}{c|}{ Middle portion } & \multicolumn{2}{c|}{ Caudal portion } \\
\cline { 2 - 7 } & $\begin{array}{c}\text { Dorso-lateral } \\
\text { region }\end{array}$ & $\begin{array}{c}\text { Ventro-medial } \\
\text { region }\end{array}$ & $\begin{array}{c}\text { Dorso-lateral } \\
\text { region }\end{array}$ & $\begin{array}{c}\text { Ventro-medial } \\
\text { region }\end{array}$ & $\begin{array}{c}\text { Dorso-lateral } \\
\text { region }\end{array}$ & $\begin{array}{c}\text { Ventro-medial } \\
\text { region }\end{array}$ \\
\hline SOM & ++ & +++ & ++ & +++ & ++++ & +++ \\
\hline NPY & +++ & ++++ & +++ & ++++ & ++++ & ++++ \\
\hline NOS & + & + & + & + & + & + \\
\hline
\end{tabular}

+ Single $;++$ Moderate $;++$ Numerous $;+++$ Very numerous

of the immunostained perikarya and fibers for each of the peptides were very similar in all the individuals studied.

1. SOM-immunoreactive cell bodies and fibers were present in the whole guinea pig dorsal striatum, but their number and density differed depending on the portion studied. Immunoreactivity was localized in the neuronal perikarya and processes (Figures 2A, $\mathrm{C}, \mathrm{E}, 3 \mathrm{~A}, \mathrm{C})$. The SOM-IR neurons were medium-sized and their cell bodies were multipolar (16-17 $\mu \mathrm{m})$, rounded (10-14 $\mu \mathrm{m})$, oval (16-17 $\mu \mathrm{m})$, fusiform $(18-22 \mu \mathrm{m})$, or triangular $(16-20 \mu \mathrm{m})$. Usually these neurons were dispersed, but some clusters of 2-4 SOM-immunoreactive neurons were found in all areas of the dorsal striatum (Figures 2A, E).

In some sections from the anterior portion of the dorsal striatum, a high density of cell bodies was observed ventro-laterally and laterally. In other sections, a high density was observed ventro-laterally and ventrally (Figures 1B, C). In this portion, rounded and multipolar perikarya predominated. In the middle of the dorsal striatum, a high density of cells was observed mostly dorso-laterally and along the lateral ventricle, but also in the cell bridges connecting the two parts of the dorsal striatum (Figures 1E, F). In this part, rounded and fusiform perikarya predominated. In the caudal portion of the dorsal striatum, the perikarya were scattered throughout (Figures 1H, I); here, oval and rounded perikarya predominated.
SOM-IR cells showed immunoreactivity either throughout the whole perikaryon or only in part of it. Each perikarya had an oval or elongated nucleus, and 2-5 immunoreactive dendrite-like processes arose from it. These processes extended straight from their perikaryon, and could frequently be traced up to about $100 \mu \mathrm{m}$. In the area corresponding to the putamen, a small population of weakly stained neurons was found. These neurons were mainly oval to round, and possessed a relatively thin rim of cytoplasm.

Varicose SOM-IR fibers formed a network in the dorsal striatum neuropile (Figures 3A, C). Although most of these fibers were thin, thick fibers were seen sporadically (Figure 3A). The fibers were dispersed in a specific pattern throughout the structure.

In general, the density of SOM-IR fibers was higher in ventromedial regions of the dorsal striatum (Table 2). In the anterior portion of the dorsal striatum, a large mass of fibers was observed ventro-medially, just above the nucleus accumbens. In the middle of the structure, a high density of SOM-IR fibers was observed dorso-medially to the globus pallidus, just above the bed nucleus of the stria terminalis, and in the ventro-medial part of the putamen. In the caudal portion of the dorsal striatum, there was a dense network of fibers, especially in the part corresponding to the tail of the caudate nucleus; in the putamen there were far fewer fibers (Table 2). 

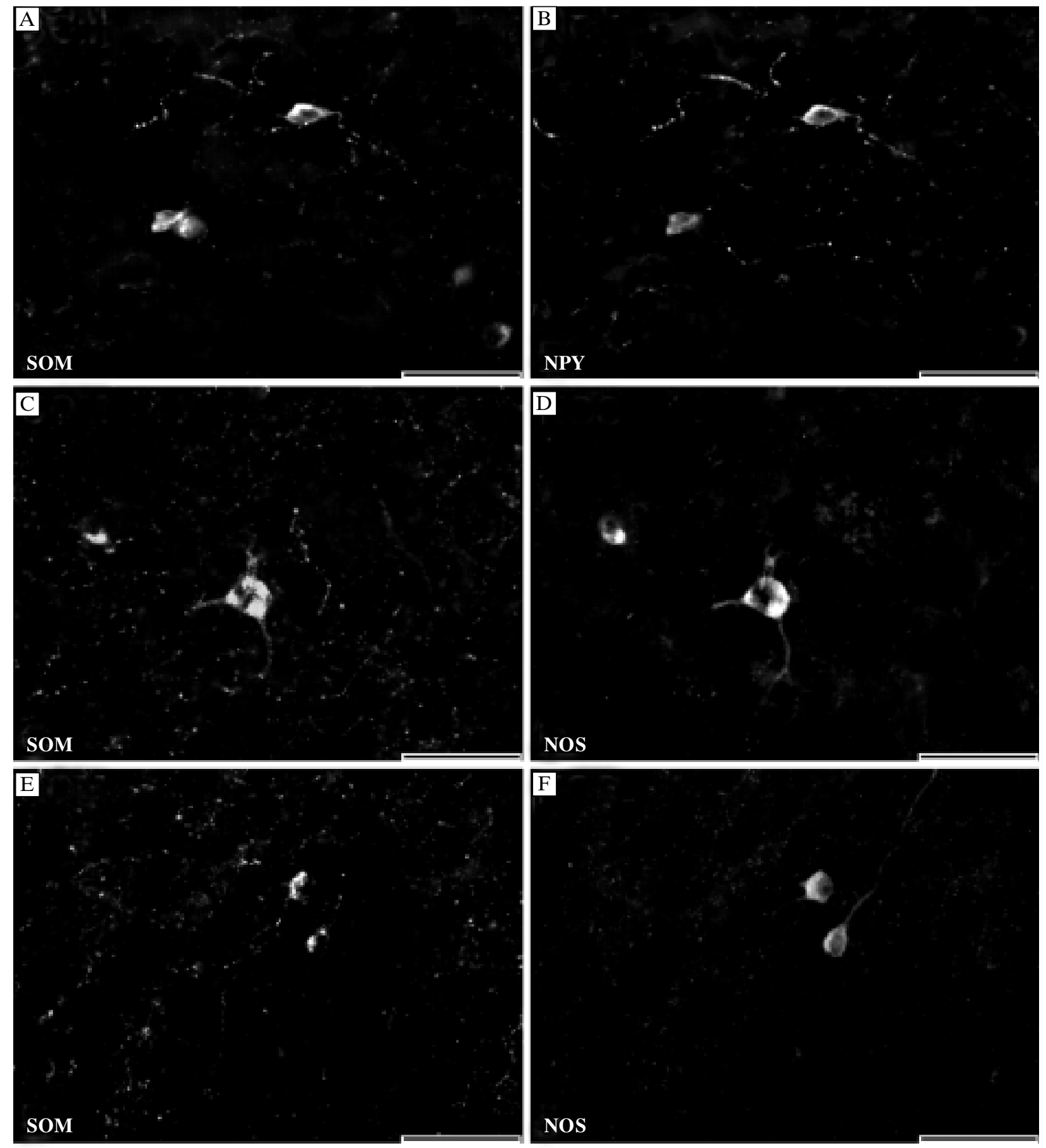

$\mathrm{F}$

Figure 2. Double-labeled neurons of SOM and NPY (A, B), SOM and NOS (C-F) in the guinea pig dorsal striatum. Note the presence of clusters of double-labeled cells (A, B, E, F). Scale bars: $50 \mu \mathrm{m}$

2. Throughout the entire rostral-caudal extent of the dorsal striatum, NPY-IR cells were distributed in a pattern that resembled the pattern of SOM-IR cells (Figures 1B, E, H). The shapes and sizes of NPY-IR cell bodies resembled those of SOM-IR cells, except in the caudal portion of the dorsal striatum, where fusiform cell bodies predominated. NPY-IR cell bodies often contained large, oval nuclei (Figure 2B). The cell bodies clustered in groups of 2-4 cells, like SOM-IR cell bodies (Figure 2F).

The morphology of NPY-IR fibers was very similar to those of SOM-IR fibers; both thick and thin fibers were observed (Figures 3A-B). NPY-IR fibers were significantly more numerous than SOM-IR fibers (Table 2). In the middle portion of the dorsal striatum, a high density of NPY-IR fibers was additionally observed along 

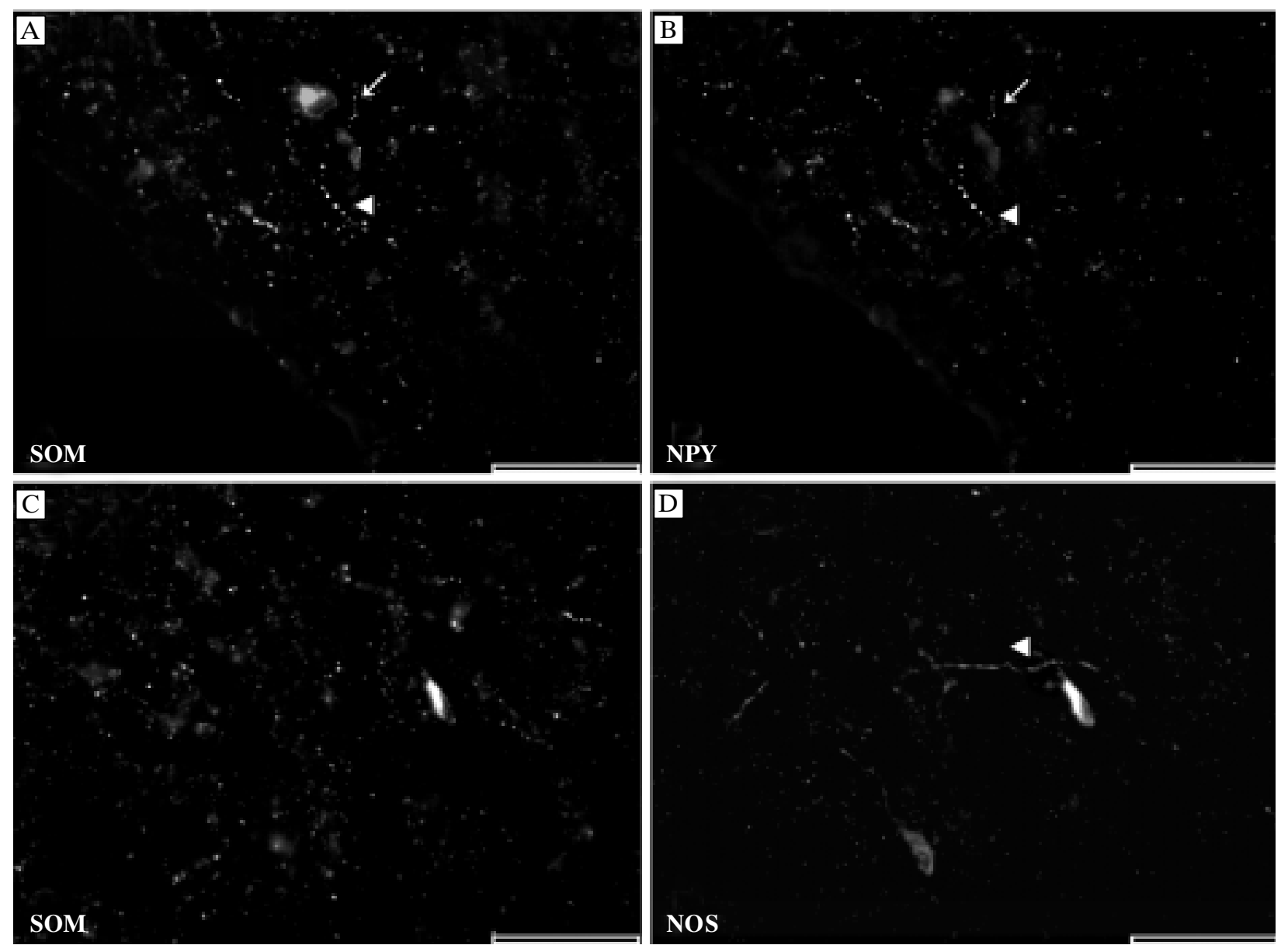

Figure 3. Examples of the co-expression of SOM and NPY (A, B), as well as SOM and NOS (C, D) in nerve fibers within the guinea pig dorsal striatum. Short arrows indicate examples of thick fibers, and long arrows indicate examples of thin fibers. Note a lack of co-localization of SOM and NOS in nerve fibers (C, D). Scale bars: $50 \mu \mathrm{m}$

the lateral ventricle, especially in the vicinity of the globus pallidus. In the caudal portion, in the part corresponding to the tail of the caudate nucleus, the distribution of NPY-IR fibers resembled that of SOM-IR fibers. A particularly dense network of NPY-IR fibers was also found in the part corresponding to the putamen.

3. NOS-IR structures were distributed similarly to SOM-IR and NPY-IR ones (Figures 1B, C, E, F, $\mathrm{H}, \mathrm{I}, 2 \mathrm{C}-\mathrm{F})$. The highest density of NOS-IR perikarya was found in the same parts of the dorsal striatum as SOM or NPY-IR cells, with one exception. NOS-immunoreactivity mainly concerned the lateral region of the dorsal striatum (Figures $1 \mathrm{C}, \mathrm{F}, \mathrm{I})$. Especially in the caudal part of the structure, NOS-IR perikarya were observed in the dorso-lateral and ventro-lateral parts of the putamen. The morphology of NOS-IR perikarya was very characteristic, with large, distinct, oval and multiindented nuclei (Figure 2F).

Where there was a distinct border between the caudate nucleus and putamen, the rounded and fusiform
NOS-IR cell bodies predominated, as in SOM-IR cells in that region. In the caudal portion of the dorsal striatum, multipolar NOS-IR neurons were present in the area that corresponds to the caudate nucleus, and rounded and fusiform neurons in the area that corresponds to the putamen.

There were also two types of NOS-IR fibers: thick and thin. The morphology of thin NOS-IR fibers was different from that of SOM- or NPY-IR fibers; these NOS-IR fibers had tiny, irregularly spaced varicosities, and they were even thinner than other fibers (Figure 3D). Thin NOS-IR fibers predominated. Thick NOS-IR fibers were only seen occasionally; they differed from SOM-IR and NPY-IR ones in that they were smooth (Figure 3D). On the analyzed sections, unlike SOM and NPY-fibers, the NOS-containing fibers did not show differences between the dorsal and ventral parts of sections (Table 2). A low expression of staining for NOS-IR fibers was seen throughout the entire dorsal striatum (Figure 3D). 


\section{Coexistence pattern of SOM and NPY, NOS in the guinea pig dorsal striatum}

All the details about the coexistence of SOM with NPY and with NOS in the dorsal striatum, as well as the morphology and characteristic features of single- and double-labeled immunoreactive elements, are shown in Figures 1B, C, E, F, H, I and 2 and 3 and Tables 1.

The most important observations were:

1. The co-existence both of SOM and NPY and of SOM and NOS in the cell bodies was confirmed (Figures 1B, C, E, F, H, I, 2A-F). However, some SOM-IR neurons did not show NPY-IR or NOS-IR immunoreactivity. Clusters of 2-4 double-labeled cells were observed in the dorsal striatum (Figures $2 \mathrm{~A}, \mathrm{~B}, \mathrm{E}, \mathrm{F}$ ).

2. In some thick and some thin fibers, SOM and NPY were co-expressed; and in the other fibers, they were not co-expressed (Figures 3A, B). SOM and NOS did not co-express in any fibers (Figures 3C, D).

3. Nearly $100 \%$ of SOM-IR neurons in the dorsal striatum contain NPY and NOS, and nearly $100 \%$ of NPY neurons contain SOM. However, only $90 \%$ of NOS-IR neurons contain SOM (Table 1).

\section{Discussion}

This is the first study to provide such a detailed description of the distribution and neurochemical characteristics of SOM-IR structures in the dorsal striatum of the guinea pig.

The distribution of SOM-IR cell bodies and fibers in the guinea pig dorsal striatum is similar to that found in previous studies with rats [3, 12, 14, 31, 32], hedgehogs and sheep [17], cats, monkeys [19] and humans [21]. SOM-immunoreactive neurons and fibers are present throughout the dorsal striatum of the guinea pig.

In the guinea pig, labeled neurons usually appear singly in the dorsal striatum. Occasionally, they form clusters in the guinea pig (present results) as well as in the rat and the human $[21,31]$. In this study, the average size of SOM-IR neurons in the dorsal striatum of the guinea pig was similar to their size in rats and cats $[16,31]$.

The morphology of SOM-IR neurons indicate that most of them belong to the medium-sized aspiny interneurons $[3,14,33]$ that correspond to the aspiny type I and III cells of DiFiglia et al. [34] and to the aspiny type III cells of Wasilewska et al. [35].

In this study, the populations of well-stained and weakly stained SOM-IR neurons were found in the dorsal striatum. The population of weakly stained neurons was found in the area corresponding to the putamen. These neurons were mainly small, oval to rounded, and possessed a relatively thin rim of cytoplasm. This kind of SOM-positive neuron has been observed by Vincent et al. [22] and DiFiglia et al. [31]. In our study, the population of weakly stained SOM-IR neurons is much less numerous than that of Vincent et al. [22]; most of the cells we observed show NPY-immunoreactivity.

In our study, a high density of SOM-positive perikarya was observed just above the nucleus accumbens. The region of nucleus accumbens may receive SOM-NPY afferents and SOM and NPY may play a role in the modulation of motor activity [36]. The second place in which numerous perikarya were seen was just above the bed nucleus of the stria terminalis, near the globus pallidus. In the rat, the striatal afferent neurons that contain SOM may originate in the area of the globus pallidus [37]. The medium-sized, spiny interneurons that contain SOM/NPY contact the medium-sized spiny neurons through symmetrical synapses and non-synaptic junctions [38].

In the guinea pig, there were mostly thin SOM-IR fibers and some thick SOM-IR fibers. In the squirrel monkey, a moderately dense and highly heterogeneous network of SOM-positive fibers was observed throughout the dorsal striatum [18]. In the rat, very small diameter unmyelinated SOM-positive fibers were also seen coursing throughout the caudate neuropile [31]. They formed swellings and made synapses en passant [31]. In the guinea pig, the density of SOM-IR and NPY-IR fibers is higher in ventromedial regions of the dorsal striatum. A similar pattern of fiber distribution was described by Kowall et al. in humans [21] and by Vuillet et al. in rats [39]. In the guinea pig, a high density of fibers containing SOM and NPY was also seen in the part corresponding to the tail of the caudate nucleus. In humans, increased concentrations of SOM and NPY were observed in the tail of the caudate and the posterior putamen by Beal et al [36].

The present study shows that most SOM-IR neurons in the dorsal striatum of the guinea pig also contain NPY-IR and NOS-IR. Nearly $100 \%$ of SOM-IR neurons in the dorsal striatum contain NPY and NOS, and nearly $100 \%$ of NPY neurons contain SOM. However, only $90 \%$ of NOS-IR neurons contain SOM. In other species, the extent of co-localization appears to be in dispute. In monkeys, extensive but incomplete co-localization of SOM and NPY has been reported [19]. All striatal SOM-IR neurons (99\%) were observed to contain NPY, but only about $85 \%$ of striatal NPY-IR neurons were found to contain SOM [19]. In the dorsal striatum of the rat, Rushlow et al. [14] reported that the population of SOM-pos- 
itive neurons is not homogeneous: $16 \%$ of SOM-IR striatal neurons are devoid of NPY, but all neurons that contain NPY also contain SOM. However, Figueredo-Cardenas et al. [9] reported that $22 \%$ of neurons that contain SOM do not contain NPY in the dorsal striatum of the rat.

In the dorsal striatum of the rat, all SOM-immunoreactive cells showed NOS-immunoreactivity and all NOS cells showed SOM-immunoreactivity [40]. In humans, SOM-immunoreactivity and nicotinamide adenine dinucleotide phosphate diaphorase (nitric oxide synthase) activity were completely co-localized [41]. In this study, nearly $10 \%$ of NOS-IR neurons did not contain SOM. Incomplete SOM/NOS co-localization was reported by Figueredo-Cardenas et al. [9] in the rat.

The differences between the results of different authors probably reflect the sensitivity of the antiserum used. In the present study, we used antisera selective for SOM 14 to detect SOM-IR neurons, which is only detectable in a subpopulation of the striatal neurons synthesizing prosomatostatin [14].

SOM may have several roles in the dorsal striatum which are related to both afferent and intrinsic systems [31]. SOM-containing afferents to the striatum may originate from limbic system nuclei, which project in a similar distribution [42]. The corticostriatal pathway influences the expression of SOM at a translational, a processing, or a metabolic level in a topographically restricted population of striatal SOM-containing neurons [32].

The peptide SOM can modulate the functional output of the basal ganglia $[43,44]$. In the striatum, SOM is produced by intrinsic interneurons, the axons and dendrites of which are selectively concentrated in the major striatal compartment, the matrix [5]. The axons of SOM-positive cells in the striatum may extend up to $1 \mathrm{~mm}$ from the cell body, which allows these cells to influence other neurotransmissions and the flow of blood in a larger space than the other striatal interneurons do $[3,5,39,45]$. It is possible that some of the SOM neurons may have axons projecting to the globus pallidus [31].

SOM/NOS-positive cells receive direct cortical impulses [45], and cholinergic [39] and dopaminergic innervation [45]. NOS-positive cells are likely to control the local flow of blood in the striatum in response to cortical and pallidal impulses [46].

SOM acts presynaptically to regulate GABA release from the terminals interconnecting mediumsized spiny projection neurons. Because SOM is released by a class of local interneuron, it has been concluded that this neuron helps to regulate the selection of motor acts [47].
The activity of striatal cholinergic neurotransmission is modulated by SOM [48]. SOM inhibits the release of ACh in slices of rat caudate nucleus, and this release is mediated by dopaminergic mechanisms [ 49 , 50]. SOM-IR neurons contain NPY [9], they receive direct glutamatergic cortical inputs [45] and they could influence other neurotransmitters such as dopamine (DA) [51, 52] and serotonin [53].

In the dorsal striatum, the neuropeptide SOM stimulates dopamine levels both in vitro [52] and in vivo [54]. In vivo, the SOM-stimulated release of DA is mediated by a glutamate-dependent action [55]. In patients with Huntington's disease, SOM is markedly elevated in the dorsal striatum [56, 57]; in patients with schizophrenia, SOM is attenuated in the cerebral cortex and the cerebrospinal fluid $[58,59]$. In the striatum, SOM has been found to increase the in vivo release of DA [55] and ACh [48]. SOM is able to release in vivo both ACh and DA, but the stimulation of ACh release by ACh release-stimulating effect of SOM is masked unless the tonic inhibitory action of endogenous DA is attenuated. Thus, in schizophrenia, where the dopaminergic hyperactivity is obvious, SOM may be involved in producing cholinergic balance [60].

SOM is a multifunctional peptide involved in several neurodegenerative diseases [23]. Kumar [23] suggests that the presence of SOM in interneurons causes these neurons to resist excitotoxicity and also provides neuroprotection to the surrounding neurons once released in the presence of excitotoxins [23]. A subclass of medium-sized aspiny interneurons that co-localize SOM, NPY, and NADPH-d survive the neuro-degenerative process [61-63].

Striatal SOM receptors (sst(1), sst(2) and sst(4)) are involved in locomotor activity [64]. The number of SOM receptors has been found to increase in patients suffering from Parkinson's disease [65, 66].

In conclusion, this study has shown that the large population of SOM-IR neurons co-transmits with NPY or NOS. This may be useful in interpreting studies on the effects of neurodegenerative diseases, excitotoxins and other experimental manipulations on the survival of striatal neurons containing SS, NPY and/or NOS.

\section{References}

1. Brazeau P, Vale W, Burgus R et al. Hypothalamic polypeptide that inhibits the secretion of immunoreactive pituitary growth hormone. Science. 1973;179:77-79.

2. Finley JC, Grossman GH, Dimeo P, Petrusz P. Somatostatin-containing neurons in the rat brain: widespread distribution revealed by immunocytochemistry after pretreatment with pronase. Am J Anat. 1978;153:483-488.

3. Johansson O, Hökfelt T, Elde RP. Immunohistochemical distribution of somatostatin-like immunoreactivity in the central nervous system of the adult rat. Neurosci. 1984;13:265-339. 
4. West MJ, Ostergaard K, Andreassen OA, Finsen B. Estimation of the number of somatostatin neurons in the striatum: an in situ hybridization study using the optical fractionator method. J Comp Neurol. 1996;370:11-22.

5. Kawaguchi Y, Wilson CJ, Augood SJ, Emerson PC. Striatal interneurons: chemical, physiological and morphological characterization. Trends Neurosci. 1995;18:527-535.

6. Cicchetti F, Prensa L, Wu Y, Parent A. Chemical anatomy of striatal interneurons in normal individuals and in patients with Huntington's disease. Brain Res Rev. 2000;34:80-101.

7. Cowan RL, Wilson CJ, Emson PC, Heizmann CW. Parvalbumin-containing GABAergic interneurons in the rat neostriatum. J Comp Neurol. 1990;302:197-205.

8. Figueredo-Cardenas G, Medina L, Reiner A. Calretinin is localized to a unique population of striatal interneurons in rats. Brain Res. 1996;709:145-150.

9. Figueredo-Cardenas G, Morello M, Sancesario G, Bernardi G, Reiner A. Colocalization of somatostatin, neuropeptide Y, neuronal nitric oxide synthase and NADPH-diaphorase in striatal interneurons in rats. Brain Res. 1996;735:317-324.

10. Holt DJ, Hersh LB, Saper CB. Cholinergic innervation in the human striatum: a three-compartment model. Neurosci. 1996;74:67-87.

11. Figueredo-Cardenas G, Anderson KD, Chen Q, Veenman CL, Reiner A. Relative survival of striatal projection neurons and interneurons after intrastriatal injection of quinolinic acid in rats. Exp Neurol. 1994;129:37-56.

12. Beal MF, Chattha GK, Martin JB. A comparison of regional somatostatin and neuropeptide $\mathrm{Y}$ distribution in rat striatum and brain. Brain Res. 1986;377:240-245.

13. Leroux P, Pelletier G. Radioautographic localization of somatostatin-14 and somatostatin-28 binding sites in the rat brain. Peptides. 1984;5:503-506.

14. Rushlow W, Flumerfelt BA, and Naus CCG. Colocalization of somatostatin, neuropeptide $\mathrm{Y}$, and NADPHd in the caudate-putamen of the rat. J Comp Neurol. 1995;351:499-508.

15. Rushlow W, Naus CC, Flumerfelt BA. Somatostatin and the patch/matrix compartments of the rat caudate-putamen. J Comp Neurol. 1996;364:184-190.

16. Chesselet MF, Graybiel AM. Striatal neurons expressing somatostatin-like immunoreactivity: evidence for a peptidergic interneuronal system in the cat. Neurosci. 1986;17:547-571.

17. Papadopoulos GC, Karamanlidis AN, Dinopoulos A, Antonopoulos J. Somatostatinlike immunoreactive neurons in the hedgehog (Erinaceus europaeus) and the sheep (Ovis aries) central nervous system. J Comp Neurol. 1986;244:174-192.

18. Desjardins C, Parent A. Distribution of somatostatin immunoreactivity in the forebrain of the squirrel monkey: basal ganglia and amygdale. Neurosci. 1992;47:115-133.

19. Smith Y, Parent A. Neuropeptide Y-immunoreactive neurons in the striatum of cat and monkey: morphological characteristics, intrinsic organization and co-localization with somatostatin. Brain Res. 1986;372:241-252.

20. Bennett-Clarke CA, Joseph SA. Immunocytochemical localization of somatostatin in human brain. Peptides. 1986;7:877-884.

21. Kowall NW, Ferrante RJ, Beal MF et al. Neuropeptide Y, somatostatin, and reduced nicotinamide adenine dinucleotide phosphate diaphorase in the human striatum: a combined immunocytochemical and enzyme histochemical study. Neurosci. 1987;20:817-828.

22. Vincent SR, von Krosigk M. Two populations of somatostatin-immunoreactive neurons in the guinea pig striatum Cell Tissue Res. 1988;252:219-222.

23. Kumar U. Somatostatin in medium-sized aspiny interneurons of striatum is responsible for their preservation in quinolinic acid and N-methyl-D-asparate-induced neurotoxicity. $\mathrm{J} \mathrm{Mol}$ Neurosci. 2008;35:345-354 [Epub 2008;May 16].

24. Albin RL, Reiner A, Anderson KD, Penney JB, Young AB. Striatal and nigral neuron subpopulations in rigid Huntington's disease: implications for the functional anatomy of chorea and rigidity-akinesia. Ann Neurol. 1990;27:357-365.

25. Beal MF, Ferrante RJ, Swartz KJ, Kowall NW. Chronic quinolinic acid lesions in rats closely resemble Huntington's disease. J Neurosci. 1991;11:1649-1659.

26. Beal MF, Kowall NW, Swartz KJ, Ferrante RJ, Martin JB. Differential sparing of somatostatin-neuropeptide $\mathrm{Y}$ and cholinergic neurons following striatal excitotoxin lesions. Synapse. 1989;31:38-47.

27 Kowall NW, Ferrante RJ, Martin JB. Pattern of cell loss in Huntington's disease. Trends Neurosci.1987;10:24-29.

28. Joosen MJ, Smit AB, van Helden HP. Treatment efficacy in a soman-poisoned guinea pig model: added value of physostigmine? Arch Toxicol. [Epub 2010;Sep 15].

29. Long YS, Huang MQ, Liao WP. Identification of novel cyclic nucleotide phosphodiesterase gene cDNAs in the brain of the guinea pig. Neurosci Bull. 2010;26:365-370.

30. Szteyn S, Gawrońska B, Szatkowski E. Topography and structure of corpus striatum in Insectivora. Acta Theriol. 1987; 32:95-104.

31. DiFiglia M, Aronin N. Ultrastructural features of immunoreactive somatostatin neurons in the rat caudate nucleus. J Neurosci. 1982;9:1267-1274.

32. Salin P, Kerkerian-Le Goff L, Heidet V, Epelbaum J, Nieoullon A. Somatostatin-immunoreactive neurons in the rat striatum: effects of corticostriatal and nigrostriatal dopaminergic lesions. Brain Res. 1990;521:23-32.

33. Nakano K, Kayahara T, Tsutsumi T, Ushiro H. Neural circuits and functional organization of the striatum. J Neurol. 2000;247:V1-V15.

34. DiFiglia M, Pasik T, and Pasik P Ultrastructure of Golgi-impregnated and gold-toned spiny and aspiny neurons in the monkey neostriatum. J Neurocytol. 1980;8:471-492.

35. Wasilewska B, Najdzion J, Robak A, Szteyn S, Bogus-Nowakowska K, Równiak M. The nerve cells of the neostriatum in the common shrew (Sorex araneus) and bank vole (Clethrionomys glareolus): a Golgi comparative study. Folia Morphol (Warsz). 2007;66:54-61.

36. Beal MF, Mazurek MF, McKee MA. The regional distribution of somatostatin and neuropeptide $\mathrm{Y}$ in control and Alzheimer's disease striatum Neurosci Lett. 1987;79:201-206.

37. Widmann R, Mensdorff-Pouilly N, Pfaller K, Sperk G. Evidence for somatostatin-containing fibers projecting from the pallidal complex to the striatum of the rat. J Neurochem. 1987;48:1857-1861.

38. Takagi H, Somogyi P, Somogyi J, Smith AD. Fine structural studies on a type of somatostatin-immunoreactive neuron and its synaptic connections in the rat neostriatum: a correlated light and electron microscopic study. J Comp Neurol. 1983; 214:1-16.

39. Vuillet J, Dimova R, Nieoullon A, Kerkerian-Le Golf L. Ultrastructural relationships between choine acetyltransferaseand neuropeptide Y-containing neurons in the rat striatum. Neurosci. 1992;46:351-360.

40. Kubota Y, Kawaguchi Y. Three classes of GABAergic interneurons in neocortex and neostriatum. Jpn J Physiol. 1994;44:145-148.

41. Selden N, Geula C, Hersh L, Mesulam MM. Human striatum: chemoarchitecture of the caudate nucleus, putamen and ventral striatum in health and Alzheimer's disease. Neurosci. 1994;60:621-636. 
42. Beal MF, Domesick VB, Martin JB. Regional somatostatin distribution in the rat striatum. Brain Res. 1983;278:103-108.

43. Allen JP, Hathway GJ, Clarke NJ et al. Somatostatin receptor 2 knockout/lacZ knockin mice show impaired motor coordination and reveal sites of somatostatin action within the striatum. Eur J Neurosci. 2003;17:1881-1895.

44. Galarraga E, Vilchis C, Tkatch T et al. Somatostatinergic modulation of firing pattern and calcium-activated potassium currents in medium spiny neostriatal neurons. Neurosci. 2007;146:537-554 [Epub 2007;Feb 26].

45. Vuillet J, Kerkerion L, Kachidian P, Boster O, Nieullon A. Ultrastructural correlates of functional relationships between nigral dopaminergic or cortical afferent fibers and neuropeptide- Y-containing neurons in the rat striatum. Neurosci Lett. 1989;100:99-104.

46. Arbuthnott GW, Kelly PAT, Wright AK. Some consequences of local blockade of nitric-oxide synthase in the rat neostriatum. In: Percheron G, McKenzie JS, Feger J (eds). Basal Ganglia IV. New York: Plenum Press; 1994:171-178.

47. Lopez-Huerta VG, Tecuapetla F, Guzman JN, Bargas J, Galarraga E. Presynaptic modulation by somatostatin in the neostriatum. Neurochem Res. 2008;33:1452-1458 [Epub 2008;Feb 13].

48. Rakovska A, Kiss JP, Raichev P, Lazarova M, Kalfin R, Milenov K. Somatostatin stimulates striatal acetylcholine release by glutamatergic receptors: an in vivo microdialysis study. Neurochem Int. 2002;40:269-275.

49. Arneric SP, Reis D. Somatostatin and cholecystokinin octapeptide differentially modulate the release of $3 \mathrm{H}$-acetylcholine from caudate nucleus but not cerebral cortex: role of dopamine receptor activation. Brain Res. 1986;374:153-161.

50. Leviel V. The reverse transport of DA: what physiological significance? Neurochem Int. 2001;38:83-106.

51. Beal MF, Martin JB. Effects of neuropeptide drugs on brain somatostatin-like immunoreactivity. Neurosci Lett. 1984;47: 125-130.

52. Chesselet M, Reisine T. Somatostatin regulates dopaminergic release in striatal slices and cat caudate nuclei. J Neurosci. 1983;3:232-236.

53. Prosperini E, Rizzi M, Fumagalli F, Tarrizo G, Samanin R, Bendotti C. Acute and chronic treatments with citalopram lower somatostatin levels in rat brain striatum through different mechanisms. J Neurochem. 1997;69:206-213.

54. Thermos K, Radke J, Kastellakis A, Anagnostakis Y, Spyraki C. Dopamine-somatostatin interactions in the rat striatum: an in vivo microdialysis study. Synapse. 1996;22:209-216.
55. Hathway G, Emson P, Humphrey P, Kendrick K. Somatostatin potently stimulates in vivo striatal dopamine and $\gamma$-aminobutyric acid release by a glutamate-dependent action. J Neurochem. 1998;70:1740-1749.

56. Aronin N, Coper P E, Lorenz LJ. Somatostatin is increased in the basal ganglia in Huntington's disease. Ann Neurol. 1983;13:519-526.

57. Stoof JC, Drukarch B, DeBoer P, Westerink BHC, Groenewegen HJ. Regulation of the activity of striatal cholinergic neurons by dopamine. Neurosci. 1992;47:755-770.

58. Molchan SE, Lawlor BA, Hill JL et al. CSF monoamine metabolites and somatostatin in Alzheimer's disease and major depression. Biol Psychiatry. 1991;29:1110-1118.

59. Rakovska AD, Kiss JP, Raichev P, Lazarova M, Kalfin R, Djambazova E. The non competitive AMPA receptor antagonist (GYKI 52466) blocks quisqualate-induced acetylcholine release from the rat hippocampus and striatum: an in vivo microdialysis study. Neurochem Int. 2002;40:419-426.

60. Rakovska A, Javitt D, Raichev P et al. Physiological release of striatal acetylcholine (in vivo): effect of somatostatin on dopaminergic-cholinergic interaction. Brain Res Bull. 2003; 61:529-536.

61. Dawbarn D, De Quidt ME, Emson PC. Survival of basal ganglia neuropeptide Y-somatostatin neurons in Huntington's disease. Brain Res. 1985;340:251-260.

62. Figueredo Cardenas G, Chen Q, Reiner A. Age dependent differences in survival of striatal somatostatin-NPY-NAD$\mathrm{PH}-$ diaphorase containing interneurons versus striatal projection neurons after intra-striatal injection of quinolinic acid in rat. Exp Neurol. 1997;146:444-457.

63. Boegman RJ, Parent A. Differential sensitivity of neuropeptide $\mathrm{Y}$, somatostatin and NADPH-d containing neurons in rat cortex and striatum to quinolinic acid. Brain Res. 1988;445,358-362.

64. Santis S, Kastellakis A, Kotzamani D, Pitarokoili K, Kokona D, Thermos K. Somatostatin increases rat locomotor activity by activating sst(2) and sst(4) receptors in the striatum and via glutamatergic involvement. NS Arch Pharmacol. 2009; 379:181-189 [Epub 2008;Sep 3].

65. Epelbaum J. Somatostatin in the central nervous system: physiological and pathological modifications. Prog Neurobiol. 1986;27:63-100.

66. Reader TA, Dewar KM. Effects of denervation and hyperinnervation on dopamine and serotonin systems in the rat neostriatum: implications for human Parkinson's disease. Neurochem Int. 1999;34:1-21.

Submitted: 31 December, 2010 Accepted after reviews: 17 March, 2011 